\title{
Gestão na atenção primária: implicações nas cargas de trabalho de gestores
}

\author{
Management in primary health care: implications on managers workloads \\ Gestión en la atención primaria: implicaciones en las cargas de trabajo de gestores
}

\section{Denise Elvira Pires de Pires ${ }^{\mathrm{a}}$ \\ Lara Vandresen ${ }^{a}$ \\ Elaine Cristina Novatzki Forte ${ }^{\mathrm{a}}$ \\ Rosani Ramos Machado ${ }^{b}$ \\ Thayse Aparecida Palhano de Melo ${ }^{a}$}

\section{Como citar este artigo:}

Pires DEP, Vandresen L, Forte ECN,

Machado RR, Melo TAP. Gestão na atenção primária: implicações nas cargas de trabalho de gestores. Rev Gaúcha Enferm. 2019;40:e20180216.

doi: https://doi.org/10.1590/19831447.2019.20180216.

\footnotetext{
- Universidade Federal de Santa Catarina (UFSC), Programa de Pós-graduação em Enfermagem. Florianópolis, Santa Catarina, Brasil.

${ }^{\mathrm{b}}$ Universidade Federal de Santa Catarina (UFSC), Departamento de Enfermagem. Florianópolis, Santa Catarina, Brasil.
}

\section{RESUM0}

Objetivo: Identificar quais aspectos da gestão na atenção primária à saúde, evidenciados na literatura atual, podem influenciar as cargas de trabalho dos gestores.

Metodologia: Revisão integrativa de literatura com coleta dos dados realizada em abril de 2016, em cinco bases de dados, com artigos publicados em inglês, espanhol e português, entre 2006 e 2016.

Resultados: Os resultados dos 78 estudos encontrados foram organizados em duas macro categorias: aumento das cargas de trabalho, especialmente pelo desafio da gestão de novo modelo de atenção e de déficits nas condições de trabalho; e redução das cargas de trabalho relacionada à capacitação das equipes e gestores, à autonomia e ao apoio aos gestores.

Conclusão: Além de identificar fatores que aumentam e diminuem as cargas de trabalho dos gestores, este estudo ilumina uma linha de investigação promissora, dada a importância da gestão para o setor saúde e para efetivação da Atenção Primária à Saúde.

Palavras-chave: Gestão em saúde. Administração de serviços de saúde. Atenção primária à saúde. Estratégia Saúde da Família. Saúde do trabalhador. Condições de trabalho.

\section{ABSTRACT}

Objective: To identify which aspects of primary health care management, evidenced in current literature, can influence manager workloads.

Methodology: Integrative literature review with data collection conducted in April 2016, in five databases, with articles published in English, Spanish and Portuguese between 2006 and 2016.

Results: The results of the 78 studies were organized into two macro categories: increased workloads, especially due to the challenge of managing a new care model and deficits in working conditions; and workloads reduction related to the training of the teams and managers, the autonomy and the support for the managers.

Conclusion: In addition to identifying factors that increase and decrease the workloads of managers, this study highlights the promising line of research, given the importance of management for the health sector and effectiveness of Primary Health Care.

Keywords: Health management. Health services administration. Primary health care. Family health strategy. Occupational health. Working conditions.

\section{RESUMEN}

Objetivo: Identificar qué aspectos de la gestión en la atención primaria de salud, evidenciados en la literatura actual, pueden influenciar las cargas de trabajo de los gestores. Metodología: revisión integradora de la literatura a través de la recolección de datos realizada en cinco bases de datos, con artículos publicados en inglés, español y portugués entre 2006 y 2016.

Resultados: Los resultados de los 78 estudios encontrados se organizaron en dos macro-categorías: aumento de las cargas de trabajo, sobre todo por el desafío de la gestión de un nuevo modelo de atención y del déficit en las condiciones laborales; y reducción de las cargas de trabajo relacionadas a la capacitación de los equipos y gestores, a la autonomía y al apoyo a los gestores.

Conclusión: Además de identificar factores que aumentan y disminuyen las cargas de trabajo de los gestores, este estudio lleva consigo una línea de investigación prometedora dada la importancia de la gestión para el sector salud y para la efectividad de la Atención Primaria de la Salud.

Palabras clave: Gestión en salud. Administración de los servicios de salud. Atención primaria de la salud. Estrategia de salud familiar. Salud laboral. Condiciones de trabajo. 


\section{—INTRODUÇÃO}

$\mathrm{Na}$ atualidade a saúde tem, sistematicamente, ocupado um lugar de destaque na preocupação das pessoas e das nações constando dentre os 17 objetivos para o desenvolvimento sustentável, aprovado pelas Nações Unidas em $2015^{\text {(1) }}$. O objetivo relativo à saúde que visa assegurar uma vida saudável e promover o bem-estar aos seres humanos em todas as idades relaciona-se diretamente com esse estudo, uma vez que implica na garantia do acesso universal e da integralidade da atenção, em todos os âmbitos de organização dos serviços.

No debate do acesso universal destaca-se a efetividade e atualidade do modelo assistencial descrito na Atenção Primária à Saúde (APS) em 1978 na Conferência de Alma Ata $^{(2)}$. O debate sobre o acesso universal tem se destacado no âmbito da Organização Mundial da Saúde e da comunidade acadêmica, e está relacionado à defesa do direito à saúde e de acesso a cuidados de qualidade. Envolve o provimento de sistemas de saúde que considerem aspectos socioculturais, organizacionais e econômicos, de modo a atender as necessidades das populações, fornecendo infraestrutura, recursos humanos e tecnologias de saúde adequadas e com custos financeiros suportáveis ${ }^{(3)}$.

Modelo assistencial envolve disponibilidade de serviços, equipamentos e instrumentos, técnicas, tecnologias e força de trabalho adequados, assim como de organização do trabalho apropriada para prestação de assistência individual ou coletiva ${ }^{(4)}$. A efetivação de modelos de atenção demanda recursos financeiros e gestão adequada. No Brasil a Política Nacional da Atenção Básica (PNAB), aprovada em 2006, atualizada em 2011 e modificada em $2017^{(5-6)}$ orienta-se pelos preceitos da APS explicitados na Estratégia Saúde da Família (ESF). Esta foi incorporada na PNAB e se propõe a reestruturar o modelo de atenção à saúde das pessoas e coletividade, orientada pelos princípios do Sistema Único de Saúde (SUS). A ESF pode ser entendida como uma inovação tecnológica não material de organização do trabalho em saúde, que visa à resolução de problemas e atendimento das necessidades de saúde de indivíduos e coletividades, articulando recursos físicos, tecnológicos e humanos ${ }^{(7)}$. Em 2017 foram aprovadas diversas mudanças significativas na PNAB(6). Estas mudanças ocorreram no contexto de um amplo processo de redução de direitos, com impacto negativo nas políticas sociais, adotadas por um governo que assume no Brasil em 2016, após destituição de uma presidente democraticamente eleita. Apesar das referidas mudanças ocorridas na PNAB, a mesma ainda se constitui na principal política pública de acesso da população aos serviços de saúde.
O modelo assistencial da APS se concretiza com interfaces entre as dimensões políticas, ideológicas, organizacionais, econômicas e culturais e, neste cenário, encontra-se o gestor das unidades locais, regionais e municipais vivendo conflitos político-ideológicos, socioeconômicos e administrativo-financeiros na busca de um agir que proporcione uma atenção à saúde em sentido ampliado e com responsabilidades mais complexas do que o estabelecido no modelo da biomedicina ${ }^{(8-9)}$.

No entanto, no caso brasileiro e em documentos das organizações multilaterais do campo da saúde ${ }^{(10)}$, apesar da complexidade envolvida nesse trabalho, não se encontra explicitação das atribuições/competências dos gestores. Além disso, a literatura que trata das implicações deste trabalho nas cargas de trabalho dos gestores é reduzida ${ }^{(11)}$.

Cargas de trabalho, neste estudo, são consideradas como elementos presentes no processo de trabalho que interatuam dinamicamente entre si e com o corpo do trabalhador, podendo desencadear desgaste e adoecimento dos trabalhadores ${ }^{(12)}$. Identificar aspectos da gestão que podem contribuir para aumentar ou reduzir as CT dos gestores pode orientar a ação política no sentido de buscar medidas para reduzir as cargas de trabalho, contribuindo para a viabilização do direito universal à saúde ${ }^{(13)}$.

Neste contexto, o presente estudo tem por objetivo identificar quais aspectos da gestão na atenção primária à saúde, evidenciados na literatura atual, podem influenciar as cargas de trabalho dos gestores.

\section{MÉTODO}

O presente estudo conforma-se em uma revisão integrativa de literatura e seguiu as etapas sugeridas por Ganong ${ }^{(14)}$, com o objetivo de compreender um fenômeno pré-determinado utilizando procedimentos reconhecidos na comunidade acadêmica para este tipo de estudo ${ }^{(15-16)}$.

Com vistas a garantir o rigor científico exigido para estudos desta natureza, o desenvolvimento metodológico seguiu as etapas estipuladas previamente em protocolo, o qual foi validado por um pesquisador externo a este estudo. O protocolo foi norteado a partir da seguinte pergunta de pesquisa: Quais aspectos da gestão na Atenção Primária à Saúde, evidenciados na literatura atual, podem influenciar as cargas de trabalho dos gestores? Em seguida, definiram-se os critérios de inclusão e exclusão, as etapas de identificação e seleção, a avaliação e a inclusão dos estudos selecionados, e por fim, a condensação dos assuntos encontrados que respondem ao objetivo deste estudo.

A coleta dos dados foi realizada por dois revisores independentes, no período de 26 a 30 de abril de 2016 nas 
bases de dados PubMed, Literatura Latino-Americana e do Caribe em Ciências da Saúde (LILACS), Scientific Electronic Library Online (SciELO), Scopus e Cumulative Index to Nursing and Allied Health Literature (CINAHL).

As chaves de busca utilizadas foram elaboradas com base nos Descritores em Ciências da Saúde (DECS) e no Medical Subject Headings (MESH), com a utilização dos operadores booleanos AND e $O R$, as quais seguem:

-Gestão em Saúde/ HealthManagement / Gestión en Salud;

-Administração em Saúde/ Health Administration/ Administración en Salud;

-Administração de Serviços de Saúde/ Health Services Administration/Administración de los Servicios de Salud;

-Atenção Primária à Saúde / Primary Health Care / Atención Primaria de Salud;

-Estratégia Saúde da Família / Family Health Strategy / Estrategia de Salud Familiar.
Adotaram-se como critérios de inclusão artigos completos que continham no título e/ou no resumo os termos estipulados nas chaves de busca, publicados e sujeitos ao acesso via VPN (Virtual Private Network), pela Universidade Federal de Santa Catarina. Também serviram de critérios de inclusão os artigos publicados nos idiomas inglês, espanhol e português entre os anos de 2006 a 2016, período este que se justifica pela busca de publicações recentes e no caso brasileiro pela adoção da Atenção Básica à Saúde como política nacional que se orienta pelos preceitos da Atenção Primária à Saúde ${ }^{(5)}$, o que continua válido, apesar das mudanças de $2017^{(6)}$.

Excluíram-se desta amostra os estudos duplicados, teses e dissertações, livros, todas as revisões de literatura, os editoriais, os boletins informativos, documentos de entidades governamentais e de resultado de eventos cientíicos, bem como artigos que se apresentaram fora da temática a ser estudada e os não disponibilizados na íntegra.

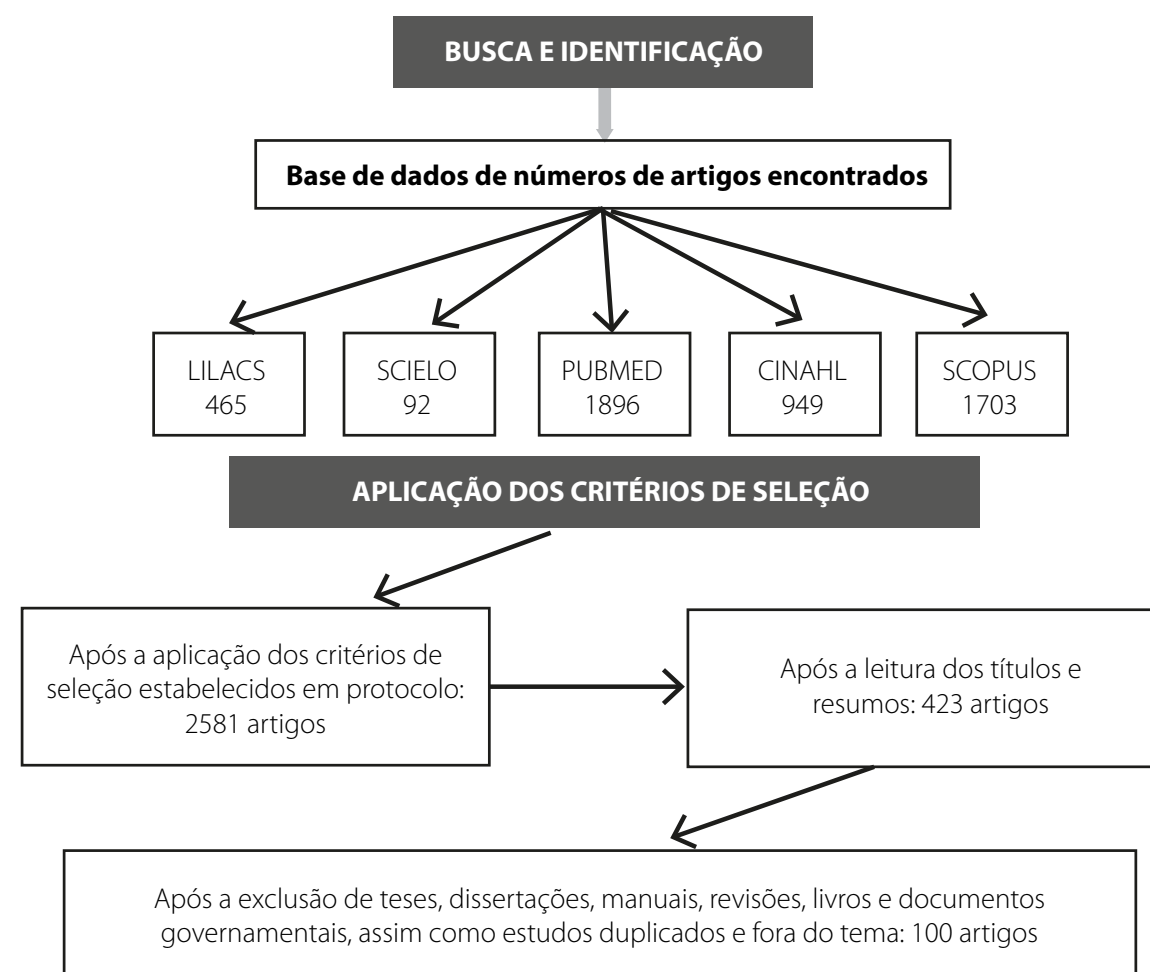

AVALIAÇÃO FINAL E INCLUSÃO

Após a compatibilização dos achados entre os cinco revisores independentes, foram selecionados para a amostra final 78 artigos

Figura 1 - Estratégia de busca e seleção dos estudos da revisão Fonte: Autores. 
O corpus da pesquisa constitui-se em 78 artigos os quais foram inseridos no software Atlas.ti (Qualitative Research and Solutions), versão 7.6.1 ${ }^{\oplus}$. A cada artigo, ao ser inserido no software, é automaticamente atribuído um número de ordem e identificado como primary document (P1, P2... sucessivamente).

O processo analítico seguiu os passos da leitura exaustiva de cada artigo a fim de identificar o conjunto do conteúdo de cada texto, após foi realizada a seleção de fragmentos significativos (quotations, na terminologia do Atlas.ti) e a atribuição de codes. A análise dos dados encontrados nos textos, e sua organização em um formato gráfico (network, linguagem do software) possibilitou sintetizar os achados e elaborar categorias analíticas.

Ressalta-se que os aspectos éticos foram observados neste artigo de revisão, citando os autores e indicando a fonte nas paráfrases.

\section{RESULTADOS}

Os achados desta revisão foram organizados em duas macros categorias: gestão na APS e aumento das CT; gestão na APS e redução das CT.

\section{Gestão na APS e Aumento das CT}

Considerando o processo de trabalho de gestor na APS, possível de apreender nos artigos publicados sobre gestão na APS, identificou-se diversos aspectos que podem contribuir para aumentar as suas cargas de trabalho, em especial o que se destacou como desafios e dificuldades encontradas no trabalho de gestão neste âmbito de atenção. 0 conjunto destes desafios e dificuldades, com a respectiva indicação da sua maior ou menor presença na literatura estudada, está apresentado na Figura 2.

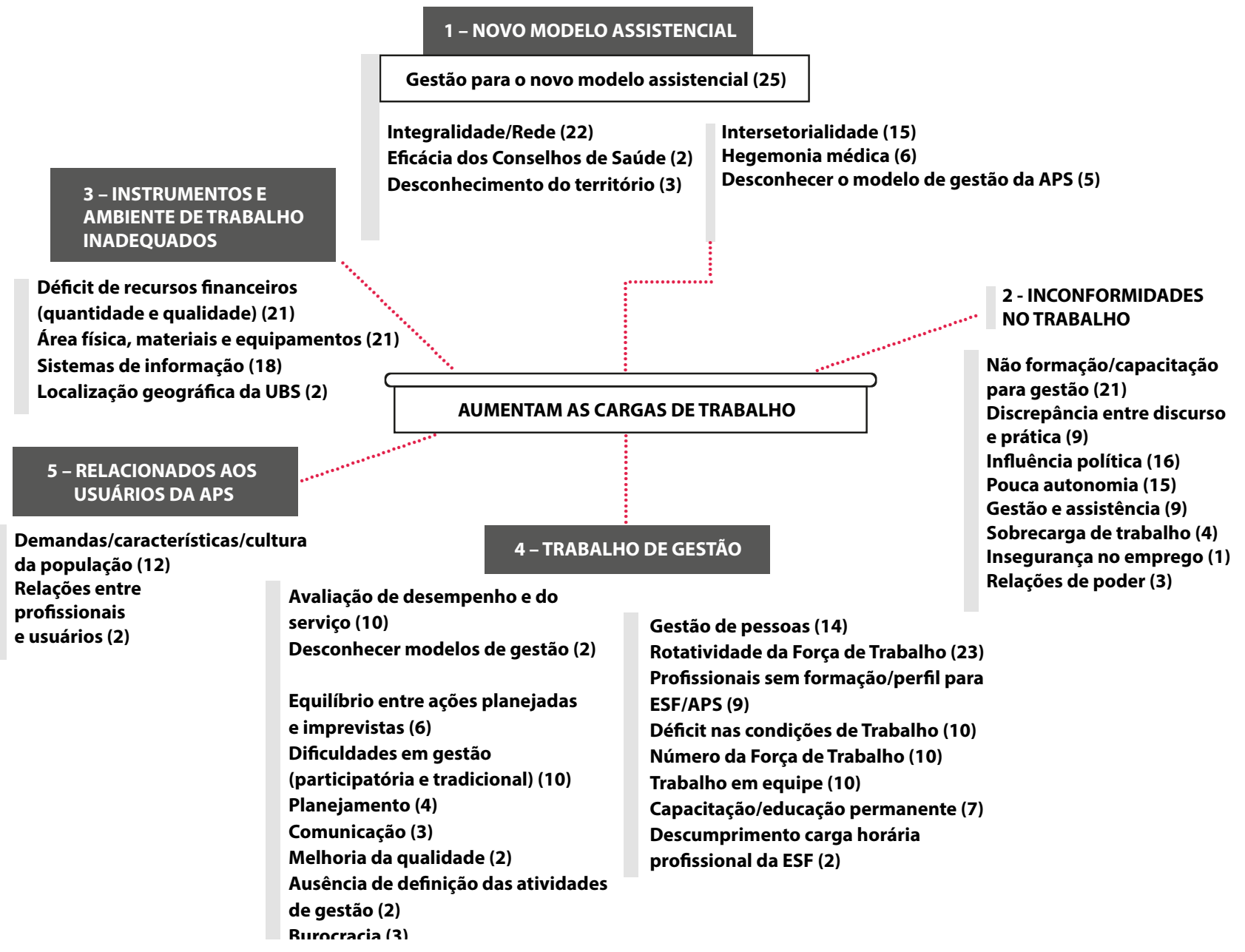

Figura 2 - Desafios e dificuldades encontradas na gestão na APS que podem aumentar as cargas de trabalho dos gestores Fonte: Autores. 
Dentre os principais desafios ou dificuldades mencionadas pelos autores que estudaram a gestão na APS, aparece, em primeiro lugar, a gestão de um novo modelo de atenção complexo, que se propõe a atender às necessidades de saúde das pessoas para além da dualidade diagnóstico-terapêutica e da atenção curativa individual, e que envolve equipes multiprofissionais orientadas para realizar um trabalho interdisciplinar ${ }^{(17-35)}$. Como ilustrado nos trechos a seguir:

A equipe de saúde deverá ter uma abordagem multidisciplinar e organizar o trabalho de forma horizontal, que possibilite, no processo decisório, a participação de todos os envolvidos. Outro atributo desta estratégia é instigar o exercício do controle social da população pela qual éresponsável(18).

Faz-se necessário um redesenho dos macroprocessos de trabalho, principalmente no que diz respeito ao entendimento de que as competências da gestão sejam ampliadas [...] e capazes de "desencadear no conjunto dos trabaIhadores um processo de reflexão e revisão de sua prática que encaminhe para a adesão e comprometimento a um processo de produção de cuidados à saúde e não de cumprimento de tarefas fragmentadas centradas no desenvolvimento de procedimentos isolados ${ }^{\prime \prime(35)}$.

Diversos aspectos da gestão, mencionados nos textos como desafios ou dificuldades, visíveis no grupo 1 da Figura 2, estão relacionados às características do próprio modelo da APS, destacando aspectos deste novo modelo que se constituem em desafio para a gestão. Dentre eles foram significativas as dificuldades de efetividade da integralidade da atenção, incluindo os déficits no funcionamento da rede de atenção ${ }^{(18-19,26,31,35-46)}$ e os desafios de implantar e fazer funcionar a intersetorialidade ${ }^{(18-19,23,25,28,31,47-53)}$. Também se identificou dificuldades relativas à eficácia dos ConseIhos de Saúde, em relação à articulação com a população para o compartilhamento e aprovação dos instrumentos de gestão do SUS, no caso brasileiro(19,54); e também dificuldades relacionadas ao desconhecimento pelos gestores do território sob sua responsabilidade ${ }^{(18,51,5)}$ e desconhecimento acerca dos aspectos que envolvem a gestão da $\operatorname{APS}^{(17,21,28,40)}$.

O segundo desafio/dificuldade que mais apareceu nos textos e que pode contribuir para aumentar as CT dos gestores é a rotatividade dos profissionais que atuam na APS (19-20,22,28,30-32,35-36,42,45-46,56,59), em especial os profissionais médicos, o que dificulta o estabelecimento de vínculo com colegas, gestores e usuários, ilustrado a seguir,
Questões como a precarização do vínculo e a falta de incentivos não estimulam a permanência dos trabalhadores nas funções, levando-os a buscar melhores oportunidades. Diversas pesquisas [...] têm demonstrado a alta rotatividade dos profissionais de saúde no Brasil, a qual gera o aumento de custos de reposição de pessoal, insatisfação no ambiente de trabalho e dificuldades no atendimento ao usuário em razão de interrupções nos serviços ${ }^{(20)}$.

A grande dificuldade que nós temos em relação aos recursos humanos é devido à rotatividade, devido ao perfil também, sabe? Isso dificulta o andamento do trabalho ${ }^{(35)}$.

A rotatividade dos profissionais está associada a outros aspectos relacionados ao fazer típico da gestão destacando-se a gestão de pessoas, ou seja, a gestão da força de trabalho em saúde ${ }^{(17,19-20,27,31,36,45-46,51,59,60-63)}$, sua capacitação para atuar na APS, as condições de trabalho que a mesma está submetida $(19,36,40-42,49)$, o funcionamento e efetividade do trabalho das equipes que atuam na APS, todos apresentados no grupo 4 da Figura 2. Neste grupo, também integrando o fazer dos gestores, identificou-se aspectos relacionados ao planejamento, aos modos de organização do trabalho, à gestão da qualidade, dentre outros.

E, em terceiro lugar destacaram-se aspectos descritos nos grupos 2 e 3 da Figura 2.

No grupo 2 estão descritos aspectos relacionados a força de trabalho do gestor incluindo: os déficits na capacitação para a gestão(18,20,27,36,40,42,47,64-69), discrepância entre discurso e prática ${ }^{(70)}$ influência política ${ }^{(17,19,36,38,40,44,61,63,66-67)}$; pouca autonomia ${ }^{(17,25,29,36,40,50,56,59,63,66,68)}$; sobrecarga de trabaIho ${ }^{(22,36,55,66)}$; duplicidade de atividades - gestão e assistência, especialmente no caso dos enfermeiros; insegurança no emprego ${ }^{(17,22,26,40,45,48,57)}$ e dificuldades relativas a relações de poder.

Citações relativas ao déficit de capacitação para gestão estão ilustradas a seguir:

[...] gestores com pouca formação em Saúde Pública e pequena experiência prévia em gestão(40).

A maior parte dos dirigentes dos municípios selecionados vem adquirindo, na prática, a experiência necessária ao trabalho de gestão. Também sugerem carência de aperfeiçoamento profissional, já que a maioria dos entrevistados não tinha feito cursos que oferecessem suporte ao desempenho das tarefas de gestão(31).

Essa falta de formação para a gestão e para a gestão na APS afeta de forma significativa a efetividade do modelo de atenção proposto, no qual o gestor tem o papel de coordenação com vistas a oferecer uma atenção centrada nos 
usuários, os quais possuem necessidades assistenciais cada vez mais complexas.

No grupo 3 estão descritos aspectos relacionados aos instrumentos de trabalho, entendidos em sentido amplo e incluindo déficits na área física, equipamentos, materiais e sistemas de informação(18,24,27,38,41-42,44-46-50,54,57,61-62,64-66), problemas na localização geográfica das unidades locais de saúde ${ }^{(65,71)}$, com destaque para os déficits no financiamento e também de eficiência na aplicação dos recur$\operatorname{sos}^{(18-19,24,27-28,30,35-36,40,50,65,71-73)}$.
Também foram identificados aspectos relacionados a quem se dirige o trabalho em saúde, ou seja, os usuários dos serviços como possíveis geradores de aumento das cargas de trabalho dos gestores $(17,19,24,27,35,40,50,65-66,70)$, estes aspectos integram o grupo 5 da Figura 2.

\section{Gestão na APS e Redução das CT}

Aspectos que facilitam a gestão na APS e que podem contribuir para reduzir as cargas de trabalho dos gestores, encontrados nesta revisão, estão apresentados na Figura 3.

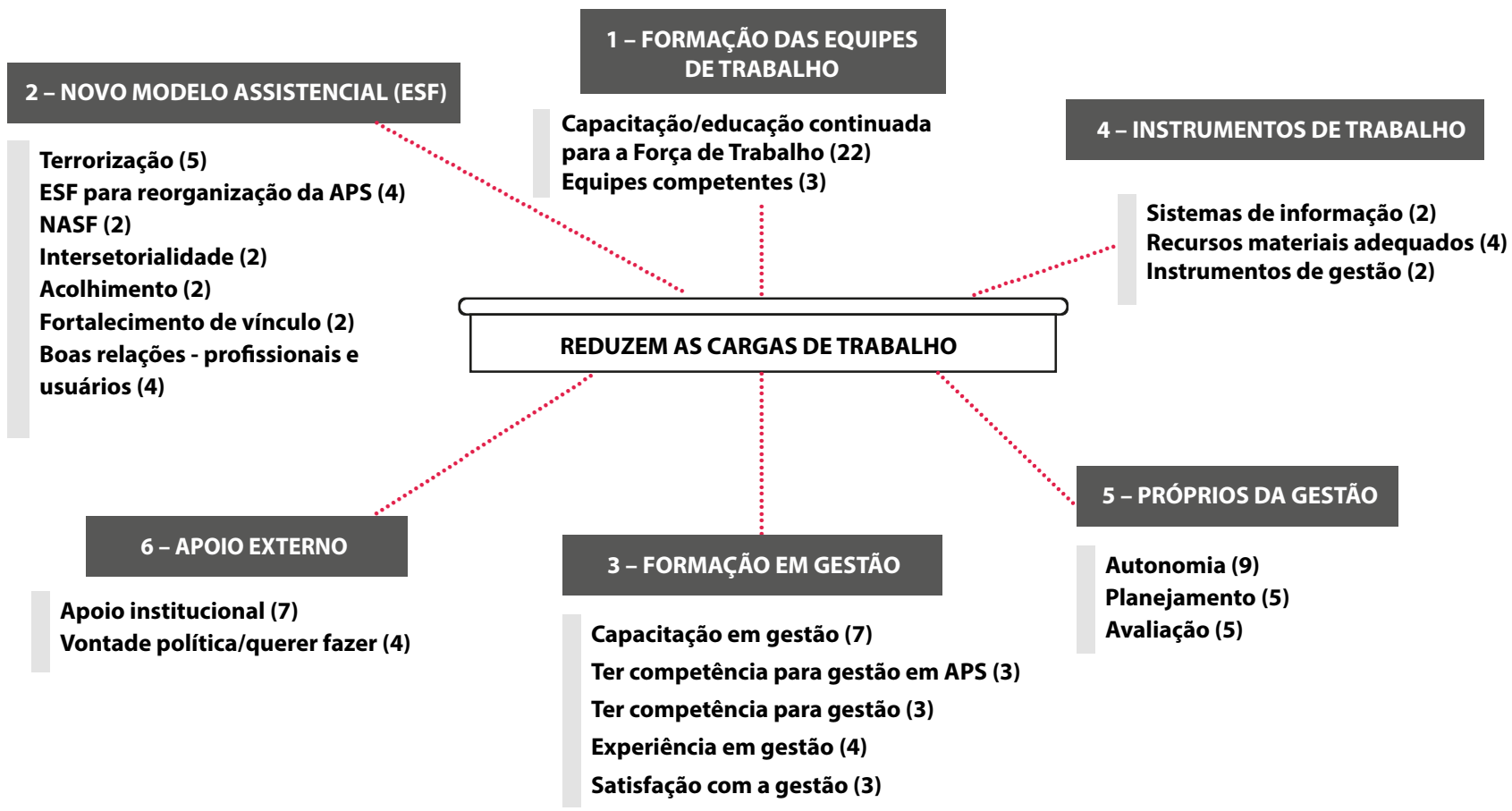

Figura 3 - Aspectos da gestão na APS que podem contribuir para reduzir as cargas de trabalho dos gestores. Fonte: Autores.

Os resultados indicam que a capacitação/educação continuada da força de trabalho, das equipes que atuam na APS, é um dos principais fatores que contribui para reduzir as cargas de trabalho dos gestores $(19-25,27,32,34,47-48,51,56,61,74-76)$, aspecto descrito no grupo 1 da Figura 3.

A importância da educação/capacitação para a gestão e da experiência em gestão também foram identifi$\operatorname{cadas}^{(17,27,56,76)}$, elementos descritos no grupo 3 da Figura 3. A literatura nacional e internacional é convergente em relação a importância da capacitação/educação dos gestores, o que pode contribuir para a redução das cargas dos mesmos. Um estudo menciona que gestores/gerentes que exercem cargos de alta, média gerência ou outra, necessitam estar inseridos no processo de educação permanente ${ }^{(36)}$. Outro estudo refere que na Sérvia o déficit de competência percebido no gerenciamento de saúde pode ser reduzido, significativamente, através do treinamento(75). Outros ${ }^{(77-80)}$ mostram, ainda, que a capacitação dos gestores pode reduzir as cargas de trabalho, ao facilitar os processos no gerenciamento das unidades de saúde.

Os grupos 5 e 6 articulam aspectos típicos do trabalho de gestão, do modo de realizá-lo e das condições institucionais disponíveis. 
Dentre eles destacou-se a questão da autonomia desses gestores, grupo 5, como um dos fatores que contribuem para reduzir as cargas de trabalho $225-27,42,56,60-61,76)$. A autonomia responsável, na prática dos gerentes, é de fundamental importância para a tomada de decisões no gerenciamento do território. Para os gestores significa a priorização das ações a serem desenvolvidas pela equipe durante o cuidado aos sujeitos, famílias e comunidade ${ }^{(75)}$. A autonomia mínima necessária para o desempenho adequado de suas funções indica a necessidade do gestor em atuar em favor de transformações e ter vontade política para buscá-las ${ }^{(42)}$, além de exercer a liderança ${ }^{(81)}$, investir no planejamento ${ }^{(82)}$ e na territorialização(83), fazer uso dos sistemas de informação disponíveis ${ }^{(84-86)}$ e melhorar a comunicação(87).

O apoio institucional é outro dos fatores, descrito no grupo 6, como facilitador para a gestão e que pode contribuir para redução das cargas de trabalho dos gestores. Apoio institucional a ser ofertado a partir das necessidades que o serviço ou grupo apresenta. O papel do apoiador é ser prudente, estar atento às necessidades do grupo, ouvir as angústias, fragilidades e fortalezas, interceder nos momentos de conflitos, na tentativa de possibilitar um ambiente de confiança e solidariedade ${ }^{(21)}$. Estudos internacionais ${ }^{(36,71,88-89)}$ corroboram com os brasileiros, quando destacam mudanças no processo de trabalho e que essas mudanças ocorreram em função do apoio(21,25,37,66,90-91), sinalizando para uma gestão mais participativa, onde todos são responsabilizados e possuem espaços coletivos deliberativos. O apoio é apresentado como precursor da mudança no modo de conduzir a gestão ${ }^{(37)}$. No grupo 5 encontra-se, ainda, que a realização de planejamento e de avaliação também são aspectos que contribuem para a redução das cargas de trabalho dos gestores na APS $113,56,61,64,92)$. A avaliação dos serviços e a criação da Estratégia Saúde da Família, esta última no Brasil, apareceram como fatores que contribuem para reorganização da APS e para a redução das cargas de trabalho dos gestores na AB/APS(20,92-94).

Ainda se encontrou outro grupo de elementos, grupo 2 na Figura 3, que dizem respeito as próprias características do novo modelo assistencial da APS ${ }^{(35,37,49,65)}$. Estes elementos, quando presentes e realizados adequadamente, contribuem para reduzir as $C T$ dos gestores.

\section{DISCUSSÃO}

O estudo mostrou a existência de literatura significativa sobre gestão na APS, sendo possível identificar diversos aspectos, incluindo dificuldades e facilidades presentes no trabalho do gestor deste âmbito da assistência em saúde, que podem contribuir para o aumento e, também, para a redução das cargas de trabalho no exercício desta atividade. A gestão no setor saúde tem sido reconhecida como fundamental e um dos macroproblemas mais significativos para a efetivação das políticas públicas de saúde(95-96).

Os estudos, corpus desta pesquisa, possibilitaram identificar características do trabalho do gestor, mas nenhum tratou especificamente o que constitui esse trabalho. Também, não se encontrou nenhum artigo que tratasse das cargas de trabalho dos gestores que atuam na APS.

Destacou-se, nas publicações estudadas, a menção a desafios e dificuldades que podem gerar aumento das cargas de trabalho dos gestores, predominando a identificação da grande dificuldade de implementar um novo modelo assistencial que amplia o escopo do modelo da biomedicina.

No Brasil, a APS está incorporada na Política Nacional da Atenção Básica constituindo-se em uma inovação tecnológica do tipo não material e de organização do trabalho em saúde ${ }^{(97)}$ que amplia o escopo das ações típicas do modelo da biomedicina ${ }^{(98)}$. Isto implica em novas demandas e/ou desafios, exigindo do gestor maior responsabilização no âmbito local e participação de diversos atores, incluindo equipes capacitadas para o exercício deste trabalho, participação comunitária e investimento público. Cabe ressaltar que, no caso do Brasil, desde a implantação do Programa Saúde da Família, a proposta já era de reorganização dos serviços e reorientação das práticas de assistência. Apresentava uma nova dinâmica para a estruturação dos serviços de saúde, tendo como foco central o processo de trabalho em saúde, em uma perspectiva interdisciplinar, objetivando uma mudança no modo de tratar a saúde ${ }^{(7-8,97)}$.

Essa inovação implica em novas demandas e/ou desafios, pois exige do gestor maior responsabilização no âmbito local e participação de outros atores. Esse processo desencadeia sofrimentos com o conflito de modelos de atenção vigentes, o que pode implicar em aumento das cargas de trabalho dos gestores, os quais têm a responsabilidade de coordenar o processo. Ao gestor é requerida alta responsabilidade e coordenar múltiplos fatores sobre os quais tem fraca governabilidade.

Apesar da sinalização internacional, desde o final dos anos 1970(2), sobre a relevância do modelo da APS para a efetivação do direito à saúde das populações, ainda é muito forte a prática hegemônica voltada para a medicina curativa sustentada no paradigma da biomedicina ${ }^{(8,98)}$. Assim como é significativa a pouca clareza de gestores e/ou equipe gestora acerca do modelo de gestão que privilegia a APS como coordenadora da assistência, visando o alcance da integralidade. 
De outro lado, quando o prescrito no modelo assistencial da APS tem possibilidade de ser implementado, contribui para reduzir as cargas de trabalho dos gestores. Como é o caso da realização de ações de territorialização, de práticas intersetoriais, assim como de práticas que fortalecem o acolhimento, o vínculo e boas relações com os usuários.

Outro elemento de destaque, que parece ter forte relação com as cargas de trabalho, diz respeito à educação/formação/capacitação para a gestão e para o trabalho na APS. Déficits neste âmbito emergiram como dificultadores para a atuação do gestor na APS, destacando-se a não capacitação dos gestores para exercer este trabalho e a não capacitação das equipes para atuar neste modelo. Encontrou-se, ainda, referência à dificuldade gerada pelo acúmulo das atividades de gestão com as assistenciais, o que também é mencionado em estudo que aborda o trabalho de enfermeiros gestores na APS ${ }^{(99)}$.

A educação permanente foi identificada como essencial para a boa atuação das equipes e com efeitos positivos na redução das cargas de trabalho dos gestores. A literatura sinaliza a importância da educação permanente como estratégia para reorganização do funcionamento dos serviços e, até mesmo, dos processos de trabalho, visando a superação da alienação provocada pela sobrecarga de trabalho(100).

No Brasil existe uma política pública para educação permanente dos trabalhadores da saúde, todavia esta apresenta grande dificuldade de operacionalização, seja por recursos financeiros insuficientes, falta de vontade política ou de pessoal, ou até mesmo de capacitação das instâncias superiores para criar arranjos interinstitucionais ou setoriais para sua implementação(101). A desmotivação dos gestores para formular estratégias que articulem as necessidades de melhoria da prática destes profissionais com a educação permanente em saúde pode ser um aspecto que também dificulte a operacionalização dessa política. A articulação entre a educação e a saúde encontra-se relacionada não só às ações dos serviços de saúde, mas também, à gestão e as instituições formadoras ${ }^{(101)}$.

Destacaram-se, também, como desafios geradores de aumento das $\mathrm{CT}$ dos gestores, déficits nas condições de trabalho. Dentre eles estão os déficits na composição da força de trabalho na APS e a precarização das relações trabalhistas, com ausência de uma política salarial e de carreira, o que tem implicado em alta rotatividade dos trabalhadores, principalmente dos médicos. Estudo realizado por Carvalho e colaboradores ${ }^{(102)}$ sobre a necessidade e dinâmica da força de trabalho na Atenção Básica de Saúde no Brasil, registrou que o número de enfermeiros e médicos cadastrados nos Centros de Saúde(CS)/Unidades Básicas de Saúde (UBS) cresceram a taxas de 42\% e 17\%, enquanto que cirurgiões dentistas cresceram apenas 8\% no Brasil. Assim, apesar do crescimento do número de médicos cadastrados, a rotatividade dos mesmos é um dos aspetos que dificulta o funcionamento dos serviços e a constituição de equipes que possam atuar na perspectiva da interdisciplinaridade e da integralidade.

No âmbito das condições de trabalho, também foram significativos os déficits de recursos materiais e financeiros como possíveis geradores de aumento das cargas de trabalho do gestor, pelas dificuldades que provocam na qualidade e resolutividade da assistência aos usuários. Nesta perspectiva, a gestão na atenção primária precisa superar os limites de uma gestão parcelar e fragmentada, para um patamar em que se valorize aspectos fundamentais como condições de trabalho, política de gestão de pessoas, utilização/incorporação de tecnologias, trabalho multiprofissional, educação permanente e planejamento participativo ${ }^{(103)}$. Necessitando de gestores capazes de implantar políticas e novos modelos de atenção, mas também com capacidade e competência para gerir os graves problemas que se apresentam neste processo(104).

Também contribuem para a redução das CT dos gestores, aspectos como autonomia no trabalho, possibilidade de realizar ações de planejamento e avaliação dos resultados do trabalho, assim como contar com apoio institucional para conduzir a gestão no sentido de possibilitar a qualificação da APS e a efetivação de seus atributos e do trabalho coletivo cooperativo, mais resolutivo e satisfatório em saúde. Com relação à autonomia, um estudo publicado em 2017 sobre a coordenação das Redes de Atenção à Saúde no Rio de Janeiro, Brasil, e na região de Lisboa, Portugal, na atenção primária à saúde, mostra diferenças entre as duas cidades, com maior autonomia no Rio de Janeiro e menor na Região de Lisboa, cujos processos decisórios e planos de ação estão sob cuidados do Sistema Nacional Português. Autonomia nos processos decisórios tem relação com decisões políticas ou institucionais, interferindo na ação dos gestores ${ }^{(105)}$.

O planejamento e a avaliação são destacados nos estudos como contribuintes para a organização do trabalho na APS e como essenciais para uma gestão adequada, todavia a escolha do método de planejamento interfere nos resultados. E, além do método, a definição dos problemas ou diagnóstico situacional constituem-se em ponto de partida para realização do planejamento(103). Cabe destacar ainda que as metas impostas pela macro política do sistema de saúde nem sempre facilitam o processo de trabalho dos gestores e, consequentemente, das equipes na APS(106). 


\section{ロ CONCLUSÃO}

Não se encontrou na literatura estudada sobre gestão na APS uma descrição do que caracteriza o fazer do gestor neste importante âmbito de organização dos serviços de saúde. No entanto, encontrou-se número significativo de publicações sobre gestão na APS, mostrando que a mesma é fundamental para a efetivação de políticas públicas.

Também, nesta revisão, não se encontrou nenhum artigo que tratasse das cargas de trabalho dos gestores que atuam na APS. No entanto foi possível identificar, nas publicações estudadas, a menção a desafios e dificuldades que podem gerar aumento das cargas de trabalho dos gestores. E que estes foram predominantes, com destaque para a grande dificuldade de implementar um novo modelo assistencial que amplia o escopo do modelo da biomedicina, para a gestão de pessoas e para os problemas de déficit nas condições de trabalho.

Também foi possível identificar aspectos do trabalho de gestão que podem contribuir para reduzir as cargas de trabalho dos gestores. Dentre eles destacaram-se os relacionados com a disponibilidade de força de trabalho, incluindo equipes e gestores capacitados para atuar com a APS. E, ainda, o apoio institucional recebido para a operacionalização da assistência e aspectos relacionados ao gerenciamento das ações com planejamento, autonomia e avaliação.

Para potencializar uma atenção de saúde que respeite os atributos da APS é necessário investimento na educação permanente pari passu com estratégias/políticas que minimizem os aspectos que desfavorecem o investimento/ financiamento em recursos materiais e humanos.

Estes dados sugerem que aspectos do fazer dos gestores da APS tem implicações nas cargas de trabalho dos mesmos e que este é um campo promissor de investigação podendo contribuir para ações no sentido de efetivação da APS e do acesso universal em saúde. No entanto, há necessidade de estudos com um nível maior de evidência em especial os que podem ser obtidos em pesquisas de campo.

Inclui-se, também, como limitação do estudo o corte definido para a estratégia de busca e a realização da revisão em período histórico social de mudanças significativas para a atenção primária em saúde, em especial no Brasil.

\section{口 REFERÊNCIAS}

1. Organização das Nações Unidas (BR) [Internet]. Brasilia: ONUBR; C2015-2019 [citado 2017 ago 03]. Conheça os novos 17 Objetivos de Desenvolvimento Sustentável da ONU [aprox. 1 tela]. Disponível em: https://nacoesunidas.org/ conheca-os-novos-17-objetivos-de-desenvolvimento-sustentavel-da-onu/.
2. Declaração de Alma-Ata: Conferência Internacional sobre cuidados primários de Saúde 2015 set 20 [citado 2017 ago 03]. In: OPAS Blog [Internet]. c2015- . [aprox. 1 tela]. Disponível em: https://www.opas.org.br/declaracao-de-almaata/.

3. Marziale MHP. Universal access to health and universal health coverage: nursing contributions. Rev Latino-Am Enfermagem. 2016;24:e2667. doi: https://doi. org/10.1590/1518-8345.0000.2667.

4. Paim JS. Modelos assistenciais: reformulando pensamento e incorporando a proteção e a promoção da saúde. In: Paim JS. Saúde: política e reforma sanitária. Salvador: COOPITEC/ISC; 2002. p. 367-81.

5. Ministério da Saúde (BR). Departamento de Atenção Básica. Política Nacional de Atenção Básica. Brasília, DF: Ministério da Saúde; 2012.

6. Ministério da Saúde (BR). Portaria no 2.436, de 21 de setembro de 2017. Estabelece a revisão de diretrizes para a organização da Atenção Básica, no âmbito do SUS. Brasilia: Ministério da Saúde; 2017.

7. Trindade LL, Pires DEP. Implications of primary health care models in workloads of health professionals. Texto-Contexto Enferm. 2013;22(1):36-42. doi: https:// doi.org/10.1590/50104-07072013000100005.

8. Starfield B. Atenção primária: equilibrio entre necessidades de saúde, serviços e tecnologia. Brasília: Unesco, Ministério da Saúde; 2002.

9. Portela GZ. Atenção Primária à Saúde: um ensaio sobre conceitos aplicados aos estudos nacionais. Physis. 2017;27(2):255-76. doi: https://doi.org/10.1590/ s0103-73312017000200005.

10. Organização Mundial da Saúde (CH). Relatório Mundial da Saúde 2008: cuidados de saúde primários: agora mais que nunca. Lisboa: OMS, Alto Comissariado da Saúde; 2008 [citado 2017 out 10]. Disponível em: https:// apps.who.int/iris/bitstream/handle/10665/43949/9789244563731_por. pdf?sequence $=4$ \&isAllowed $=y$.

11. Melo TAP, Pires DEP. Fatores que contribuem para as cargas de trabalho de gestores de unidades básicas de saúde. In: Vale EG, Peruzzo AS, Felli VEA, organizadores. PROENF: Programa de Atualização em Enfermagem: Gestão: ciclo 6. Porto Alegre: Artmed Panamericana; 2016. p. 123-50.

12. Laurell $A C$, Noriega M. Processo de produção e saúde: trabalho e desgaste operário. São Paulo: HUCITEC; 1989.

13. Silva RM, Andrade LOM. Coordenação dos cuidados em saúde no Brasil: o desafio federal de fortalecer a atenção primária à saúde. Physis. 2014;24(4):1207-28. doi: https://doi.org/10.1590/50103-73312014000400010.

14. Ganong LH. Integrative reviews of nursing research. Res Nurs Health 1987;10(1):1-11. doi: https://doi.org/10.1002/nur.4770100103.

15. Whittemore R, Knafl K. The integrative review: updated methodology. J Adv Nurs. 2005;52(5):546-53. doi: https://doi.org/10.1111/j.1365-2648.2005.03621.x.

16. Mendes KDS, Silveira RCCP, Galvão CM. Revisão integrativa: método de pesquisa para a incorporação de evidências na saúde e na enfermagem. Texto-Contexto Enferm. 2018 dez;17(4):758-64. doi: https://doi.org/10.1590/5010407072008000400018

17. Melo FAB, Goulart BF, Tavares DMS. Gerência em saúde: a percepção de coordenadores da estratégia saúde da família, em Uberaba-MG. Cienc Cuid Saúde. 2011;10(3):498-505. doi: https://doi.org/10.4025/cienccuidsaude. v10i3.13261.

18. Ferreira JC, Fernandes APP, Souza C, Bicudo DO, Mazza VA. A percepção do gestor sobre a organização da atenção básica à saúde da criança. Cogitare Enferm. 2010;15(1):26-32. doi: https://doi.org/10.5380/ce.v15i1.17140.

19. Heimann LS, Ibanhes LC, Boaretto RC, Castro IEN, Telesi Júnior E, Cortizo CT, et al. Atenção primária em saúde: um estudo multidimensional sobre os desafios 
e potencialidades na Região Metropolitana de São Paulo (SP, Brasil). Ciênc Saúde Coletiva. 2011;16(6):2877-87. doi: https://doi.org/10.1590/S141381232011000600025.

20. Pimenta AL. A construção de colegiados de gestão: a experiência de gestão da Secretaria Municipal de Saúde analisada por um ator político implicado. Saude Soc. 2012;21(1):29-45. doi: https://doi.org/10.1590/s010412902012000500003.

21. Maerschner RL, Bastos ENE, Gomes AMA, Jorge MSB, Diniz SAN. Apoio institucional - reordenamento dos processos de trabalho: sementes lançadas para uma gestão indutora de reflexões. Interface (Botucatu). 2014;18(Suppl 1):1089-98. doi: https://doi.org/10.1590/1807-57622013.0365.

22. Rizzotto MLF, Gil CRR, Carvalho M, Fonseca ALN, Santos MF. Força de trabalho e gestão do trabalho em saúde: revelações da avaliação externa do Programa Nacional de Melhoria do Acesso e da Qualidade da Atenção Básica no Paraná. Saúde Debate. 2014;38(spe):237-51. doi: https://doi.org/10.5935/01031104.20145018.

23. Fracolli LA, Gomes MFP, Gryschek ALFPL. Percepções de gestores municipais sobre ações de promoção da saúde: em foco os agentes comunitários de saúde. Saude Soc. 2014 [citado 2017 out 18];23(3):919-27. Disponível em: http:// www.scielo.br/pdf/sausoc/v23n3/0104-1290-sausoc-23-3-0919.pdf.

24. Pereira LP, Nery AA. Planning, management and actions of men's health in the Family health strategy. Esc Anna Nery. 2014 [cited 2017 Oct 18];18(4):63543. Available from: http://www.scielo.br/pdf/ean/v18n4/1414-8145ean-18-04-0635.pdf.

25. Casanova A0, Teixeira MB, Montenegro E. 0 apoio institucional como pilar na cogestão da atenção primária à saúde: a experiência do Programa TEIAS Escola Manguinhos no Rio de Janeiro, Brasil. Ciênc Saúde Coletiva. 2014 [citado 2017 out 18];19(11):4417-26. Disponível em: http://www.scielo.br/pdf/csc/ v19n11/1413-8123-csc-19-11-4417.pdf.

26. Ohira RHF, Cordoni Junior L, Nunes EFPA. Análise das práticas gerenciais na Atenção Primária à Saúde nos municípios de pequeno porte do norte do Paraná, Brasil. Ciênc Saúde Coletiva. 2014 [citado 2017 out 18];19(11):443948. Disponível em: http://www.scielosp.org/pdf/csc/v19n11/1413-8123csc-19-11-4439.pdf.

27. Fernandes JA, Figueiredo MD. Apoio institucional e cogestão: uma reflexão sobre 0 trabalho dos apoiadores do SUS Campinas. Physis. 2015 citado 2017 out 18]; 25(1):287-306. Disponível em: http://www.scielosp.org/pdf/physis/ v25n1/0103-7331-physis-25-01-00287.pdf.

28. Pedrosa Jl, Sousa MF, Hamann EM, Rocha DG, Oliveira MAA. Quem participa em quê? Experiências de construção compartilhada no âmbito da cooperação Brasil - Canadá para o aperfeiçoamento da gestão na Atenção Primária à Saúde. Saúde Soc. 2013 [citado 2017 out 18];22(2):629-41. Disponível em: http:// www.scielo.br/pdf/sausoc/v22n2/v22n2a30.pdf.

29. Vanderlei MIG, Almeida MCP. A concepção e prática dos gestores e gerentes da estratégia de saúde da família. Ciênc Saúde Coletiva. 2007 [citado 2017 out 18];12(2):443-53. Disponível em: http://www.scielo.br/pdf/csc/v12n2/ a21v12n2.pdf.

30. Trigueiro JVS, Nogueira JA, Sá LD, Palha PF, Villa TCS, Trigueiro DRSG. Controle da tuberculose: descentralização, planejamento local e especificidades gerenciais. Rev Latino-Am Enfermagem 2011 [citado 2017 out 18];19(6):1289-96. Disponível em: http://www.scielo.br/pdf/rlae/v19n6/pt_03.pdf.

31. Vieira JMR, Garnelo L, Hortale V A. Análise da atenção básica em cinco municípios da amazônia ocidental, com ênfase no Programa Saúde da Família. Saude Soc. 2010 [citado 2017 out 18];19(4):852-65. Disponível em: http:// www.scielo.br/pdf/sausoc/v19n4/12.pdf.

32. Ramírez NA, Romero RV. El caso de la implementación de la estrategia de Atención Primaria Integral em la Salud (APIS) en la localidad de Suba, Bogotá - Colombia. Rev Gerenc Polit Salud. 2008 [citado 2017 oct 18];7(14):125-44. Disponible en: http://revistas.javeriana.edu.co/index.php/gerepolsal/article/ view/2684/12579.

33. Silva NC, Garnelo L, Giovanella L. Extensão de cobertura ou reorganização da atenção básica? a trajetória do Programa de Saúde da Família de Manaus-AM. Saúde Soc. 2010 [citado 2017 out 18];19(3):592-604. Disponível em: http:// www.scielo.br/pdf/sausoc/v19n3/11.pdf.

34. Ronzani TM, Silva CM. 0 Programa Saúde da Família segundo profissionais de saúde, gestores e usuários. Ciênc Saúde Coletiva. 2008 [citado 2017 out 18];13(1):23-34. Disponivel em: http://www.scielo.br/pdf/csc/v13n1/06.pdf.

35. Aguilera, SLVU, França BHS, Moysés ST, Moysés SJ. Articulação entre os níveis de atenção dos serviços de saúde na Região Metropolitana de Curitiba: desafios para os gestores. Rev Adm Pública. 2013; 47(4):1021-40. doi: https://doi. org/10.1590/S0034-76122013000400010.

36. Junqueira TS, Cotta RMM, Gomes RC, Silveira SFR, Siqueira-Batista R, Pinheiro TMM, et al. As relações laborais no âmbito da municipalização da gestão em saúde e os dilemas da relação expansão/precarização do trabalho no contexto do SUS. Cad Saúde Pública. 2010 [citado 2017 out 18];26(5):918-28. Disponível em: http://www.scielo.br/pdf/csp/v26n5/14.pdf.

37. Cardoso JR, Oliveira GN, Furlan PG. Gestão democrática e práticas de apoio institucional na Atenção Primária à Saúde no Distrito Federal, Brasil. Cad Saúde Pública. 2016 [citado 2017 out 18];32(3):e00009315. Disponível em: http:// www.scielo.br/pdf/csp/v32n3/0102-311X-csp-32-03-e00009315.pdf.

38. Pinto JM, Gerhardt TE. Práticas avaliativas na gestão da Atenção Básica à Saúde: estudo de caso em Camaquã e Canguçu. Rev Adm Pública. 2013 [citado 2017 out 18];47(2):305-26. Disponível em: http://www.scielo.br/pdf/rap/v47n2/ v47n2a02.pdf.

39. Ditterich RG, Zermiani TC, Moysés ST, Moysés SJ. A contratualização como ferramenta da gestão na Atenção Primária à Saúde na percepção dos profissionais da Secretaria Municipal de Saúde de Curitiba, Brasil. Saúde Debate. 2015 [citado 2017 out 18];39(spe):207-20. Disponível em: http://www.scielo. br/pdf/sdeb/v39nspe/0103-1104-sdeb-39-spe-00207.pdf.

40. Almeida DB, Melo CMM. Avaliação da gestão na Atenção Básica nas dimensões da integralidade. Rev Baiana Saúde Publica. 2012 [citado 2017 out 18];36(3):816-30. Disponível em: http://rbsp.sesab.ba.gov.br/index.php/rbsp/ article/view/557.

41. Visagie S, Schneider M. Implementation of the principles of primary health care in a rural area of South Africa. Afr J Prim Health Care Fam Med. 2014 [cited 2017 0ct 18];6(1):E1-E10. Available from: http://www.phcfm.org/index.php/ phcfm/article/viewFile/562/821.

42. Viegas SF, Penna CMM. Implicações da integralidade na gestão municipal em saúde. Rev APS. 2013 [citado 2017 out 18];16(2):136-45. Disponível em: http://ojs2.uff.emnuvens.com.br/aps/article/view/15151.

43. Akhtar-danesh N, Valaitis R, O'Mara L, Austin P, Munroe V. Viewpoints about collaboration between primary care and public health in Canada. BMC Health Serv Res. 2013;13:311. doi: https://doi.org/10.1186/1472-6963-13-311.

44. Schimith MD, Brêtas ACP, Budó MLD, Alberti GF, Beck CLC. Gestión del trabajo: implicaciones para el cuidado en la Atención Primaria de Salud. Enferm Global. 2015 [citado 2017 oct 18];14(2). Disponible en: http://scielo.isciii.es/pdf/eg/ v14n38/administracion2.pdf.

45. Moura BLA, Cunha RC, Fonseca ACF, Aquino R, Medina MG, Vilasbôas ALQ, et al. 
Atenção primária à saúde: estrutura das unidades como componente da atenção à saúde. Rev Bras Saude Mater Infant. 2010;10(Suppl 1):s69-s81. doi: https:// doi.org/10.1590/S1519-38292010000500007.

46. Silveira DS, Facchini LA, Siqueira FV, Piccini RX, Tomasi E, Thumé E, et al. Gestão do trabalho, da educação, da informação e comunicação na atenção básica à saúde de municípios das regiões Sul e Nordeste do Brasil. Cad Saúde Pública. 2010 [citado 2017 out 18];26(9):1714-26. Disponível em: http://www.scielo. $\mathrm{br} / \mathrm{pdf} / \mathrm{csp} / \mathrm{v} 26 \mathrm{n} 9 / 05 . \mathrm{pdf}$.

47. El-Jardali F, Hemadeh R, Jaafar M, Sagherian L, El-Skaff R, Mdeihly R, et al. The impact of accreditation of primary healthcare centers: successes, challenges and policy implications as perceived by healthcare providers and directors in Lebanon. BMC Health Serv Res. 2014;14:86. doi: https://doi.org/10.1186/14726963-14-86.

48. Oliveira CM, Cruz MM, Kanso S, Reis AC, Lima A, Torres RMC, et al. Evaluability of the Program to Value Primary Healthcare Professionals (PROVAB): management challenges. Ciênc Saúde Coletiva. 2015;20(10):2999-3010. doi: https://doi. org/10.1590/1413-812320152010.13322014.

49. Cardoso AVL, Chain APN, Mendes RIP, Ferreira e Ferreira E, Vargas AMD, Martins AMEBL, et al. Assessment of the management of the Family Health Strategy via the tool Assessment for Quality Improvement in municipalities of Minas Gerais, Brazil. Ciênc Saúde Coletiva. 2015;20(4):1267-84. doi: https://doi. org/10.1590/1413-81232015204.01832014.

50. Weirich CF, Munari DB, Mishima SM, Bezerra ALQ. 0 trabalho gerencial do enfermeiro na Rede Básica de Saúde. Texto Contexto Enferm. 2009 [citado 2017 out 18];18(2):249-57. Disponível em: http://www.scielo.br/pdf/tce/v18n2/07. pdf.

51. Ruiz RRM, Acosta-Ramírez N, Rodríguez Villamizar LA, Uribe LM, León-Franco M. Experiencia de implementación de un modelo de atención primaria. Rev Salud Pública. 2011 [citado 2017 oct 18];13(6):885-96. Disponible en: https:// revistas.unal.edu.co/index.php/revsaludpublica/article/view/22897/37874.

52. Bursztyn I, Kushnir R, Giovanella L, Stolkiner A, Sterman-Heimann L, Riveros MI, Sollazzo A. Notas para el estudio de la Atención Primaria en contextos de sistemas de salud segmentados. Rev Salud Pública. 2010;12(1):77-88. doi: https://doi.org/10.1590/S0124-00642010000700006.

53. Giovanella L, Mendonça MHM, Almeida PF, Escorel S, Senna MCM, Fausto MCR, et al. Saúde da família: limites e possibilidades para uma abordagem integral de atenção primária à saúde no Brasil. Ciênc Saúde Coletiva. 2009 [citado 2017 out 18];14(3):783-94. Disponível em: http://www.scielo.br/pdf/csc/v14n3/14.

54. Berretta IQ, Lacerda JT, Calvo MCM. Modelo de avaliação da gestão municipal para 0 planejamento em saúde. Cad Saúde Pública. 2011 [citado 2017 out 18];27(11):2143-54. Disponível em: http://www.scielo.br/pdf/csp/ v27n11/08.pdf.

55. Feitosa RMM, Almeida ANS, Silva WF, Xavier MSM, Ramalho SKL, Rocha DJF. Enfermagem e gerenciamento da situação de saúde na Estratégia de Saúde da Família. Rev Baiana Enferm. 2013 [citado2017 out 18];27(2):154-63. Disponível em: https://portalseer.ufba.br/index.php/enfermagem/article/ view/6935/7159

56. Barrêto AJR, Sá LD, Silva CC, Sérgio RS, Brandão GCG. Experiências dos enfermeiros com o trabalho de gestão em saúde no estado da Paraíba. Texto Contexto Enferm. 2010 [citado 2017 out

18];19(2):300-8. Disponível em: http://www.scielo.br/pdf/tce/v19n2/11.pdf.

57. Scalco SV, Lacerda JT, Calvo MCM. Modelo para avaliação da gestão de recursos humanos em saúde. Cad Saúde Pública. 2010 [citado 2017 out 18];26(3):60314. Disponivel em: http://www.scielo.br/pdf/csp/v26n3/17.pdf.
58. Alameddine M, Saleh S, El-Jardali F, Dimassi H, Mourad Y. The retention of health human resources in primary healthcare centers in Lebanon: a national survey. BMC Health Serv Res. 2012;12:419. doi: https://doi.org/10.1186/1472-6963$12-419$.

59. Soares RS, Rau B. Gestão compartilhada: Análise e reflexões sobre o processo de implementação em uma Unidade de Atenção Primária à Saúde do SUS. Rev APS. 2009 [citado 2017 out 18];12(4):436-47. Disponível em: https://aps.ufff. emnuvens.com.br/aps/article/view/534/268.

60. Volponi PRR, Garanhani ML, Carvalho BG. Núcleo de Apoio à Saúde da Família: potencialidades como dispositivo de mudança na Atenção Básica em saúde. Saúde Debate. 2015;39(spe):221-31. doi: https://doi.org/10.5935/01031104.20155005418.

61. Fernandes LCL, Machado RZ, Anschau G0. Gerência de serviços de saúde: competências desenvolvidas e dificuldades encontradas na atenção básica. Ciênc Saúde Coletiva. 2009 [citado 2017 out 18];14(Suppl1):1541-52. Disponível em: http://www.scielo.br/pdf/csc/v14s1/a28v14s1.pdf.

62. Guimarães-X NFR, Coelho JJ. Análise do processo de trabalho dos gerentes no território da Estratégia Saúde

da Família. Rev Gerenc Polit Salud. 2012 [citado 2017 out 18];11(22):76-91. Disponível em: http://www.scielo.org.co/pdf/rgps/v11n22/v11n22a07.pdf.

63. Felisberto E, Freese E, Bezerra LCA, Alves CKA, Samico IA. Análise da sustentabilidade de uma política de avaliação: 0 caso da atenção básica no Brasil. Cad Saúde Pública. 2010 [citado 2017 out 18];26(6):1079-95. Disponível em: http://www.scielo.br/pdf/csp/v26n6/03.pdf.

64. Saliba NA, Moimaz SAS, Gonçalves PE, Lima DC, Santos JG. Gestión en salud en Brasil: la percepción de gestores y trabajadores de la salud sobre modelo de gestión y aspectos gerenciales. Acta Odont Venez. 2009 [citado 2017 oct 18];47(2). Disponible en: https://www.actaodontologica.com/ ediciones/2009/2/art-6/

65. Lima SAV, Silva MRF, Carvalho EMF, Pessoa EÂC, Brito ESV, Braga JPR. Elementos que influenciam 0 acesso à atenção primária na perspectiva dos profissionais e dos usuários de uma rede de serviços de saúde do Recife. Physis. 2015 [citado 2017 out 18];25(2):635-56. Disponível em: http://www.scielo.br/pdf/physis/ v25n2/0103-7331-physis-25-02-00635.pdf.

66. Bazzo-Romagnolli AP, Gimenez-Carvalho B, Almeida-Nunes EFP. Gestão de unidade básica de saúde em municípios de pequeno porte: instrumentos utilizados, facilidades e dificuldades relacionadas. Rev Gerenc Polit Salud. 2014 [citado 2017 out 18];13(27):168-80. Disponível em: http://www.scielo.org.co/ pdf/rgps/v13n27/v13n27a10.pdf.

67. Rocha PM, Sá AB. Reforma da Saúde Familiar em Portugal: avaliação da implantação. Ciênc Saúde Coletiva. 2011 [citado 2017 out 18];16(6):2853-63. Disponível em: http://www.scielo.br/pdf/csc/v16n6/23.pdf.

68. Castro ALB, Machado CVA. A política de atenção primária à saúde no Brasil: notas sobre a regulação e o financiamento federal. Cad Saúde Pública. 2010 [citado 2017 out 18];26(4):693-705. Disponível em: http://www.scielo.br/pdf/ csp/v26n4/12.pdf.

69. Vergara M, Bisama L, Moncada P. Competencias esenciales para la gestión en red. Rev Med Chile. 2012;140:1606-12. doi: https://doi.org/10.4067/S003498872012001200013

70. Villela WV, Araújo EC, Ribeiro SA, Cuginotti AP, Hayana ET, Brito FC, et al. Desafios da atenção básica em saúde: a experiência de Vila Mariana, São Paulo, Brasil. Cad Saúde Pública. 2009;25(6):1316-24. doi: https://doi.org/10.1590/S0102311X2009000600014

71. Mosquera PA, Hernández J, Veja R, Martínez J, Sebastián MS. Performance 
evaluation of the essential dimensions of the primary health care services in six localities of Bogota-Colombia: a cross-sectional study. BMC Health Serv Res. 2013;13:315. doi: https://doi.org/10.1186/1472-6963-13-315.

72. Nyikuri M, Tsofa B, Barasa E, Okoth P, Molyneux S. Crises and resilience at the frontline-public health facility managers under devolution in a sub-county on the Kenyan Coast. PLoS One. 2015;10(12):e0144768. doi: https://doi. org/10.1371/journal.pone.0144768.

73. Carvalho BG, Peduzzi M, Nunes EFPA, Leite FS, Silva JAM. Management of basic health units in municipalities of different size: profile and management instruments. Rev Esc Enferm USP. 2014;48(5):907-14. doi: https://doi. org/10.1590/s0080-6234201400005000018.

74. Müller EPL, Cubas MR, Bastos LC. Georreferenciamento como instrumento de gestão em unidade de saúde da família. Rev Bras Enferm. 2010;63(6):978-82. doi: https://doi.org/10.1590/S0034-71672010000600017.

75. Santric-Milicevic MM, Bjegovic-Mikanovic VM, Terzic-Supic ZJ, Vasic V. Competencies gap of management teams in primary health care. Eur J Public Health. 2011;21(2):247-53. doi: https://doi.org/10.1093/eurpub/ckq010.

76. Ximenes Neto FRG, Sampaio JJC. Gerentes do território na Estratégia Saúde da Família: análise e perfil de necessidades de qualificação. Rev Bras Enferm. 2007;60(6):687-95. doi: https://doi.org/10.1590/5003471672007000600013

77. Sales NC, Silva MGC, Pinto FJM. Competências essenciais desenvolvidas por coordenadores de Centros de Saúde da Família. Rev Bras Promoç Saúde. 2014;27(3):389-97. doi: https://doi.org/10.5020/18061230.2014.p389.

78. Araújo CEL, Pontes RJS. Constituição de sujeitos na gestão em saúde: avanços e desafios da experiência de Fortaleza (CE). Ciênc Saúde Coletiva. 2012;17(9):235765. doi: https://doi.org/10.1590/S1413-81232012000900016.

79. Magnago C, Pierantoni CR. Dificuldades e estratégias de enfrentamento referentes à gestão do trabalho na Estratégia Saúde da Família, na perspectiva dos gestores locais: a experiência dos municípios do Rio de Janeiro (RJ) e Duque de Caxias (RJ). Saúde Debate. 2015;39(104):9-17. doi: https://doi. org/10.1590/0103-110420151040194.

80. Rolim-Ensslin S, Dutra A, Souza-Duarte C, Bortoluzzi SC, Ripoll-Feliu VM. A avaliação de desempenho como proposta para gestão das equipes do programa brasileiro "Estratégia da Saúde da Família". Rev Gerenc Polit Salud. 2014 [citado 2017 out 18];13(26):10-25. Disponível em: http://www.scielo.org.co/pdf/ rgps/v13n26/v13n26a02.pdf.

81. Melo RC, Machado ME. Coordination of family healthcare units done by nurses: challenges and potential. Rev Gaúcha Enferm. 2013;34(4):61-7. doi: https:// doi.org/10.1590/S1983-14472013000400008.

82. Melo LMF, Martiniano CS, Guimarães J, Souza MB, Rocha PM. Análises das diretrizes para 0 apoio institucional das gestões da Atenção Básica das capitais brasileiras. Saúde Debate. 2016;40(108):8-22. doi: http://dx.doi. org/10.1590/0103-1104-20161080001.

83. Goldstein RA, Barcellos C, Magalhães MAFM, Francisco GRV. A experiência de mapeamento participativo para a construção de uma alternativa cartográfica para a ESF. Ciênc Saúde Coletiva. 2013;18(1):45-56. doi: https://doi. org/10.1590/S1413-81232013000100006.

84. Bittar TO, Meneghim MC, Mialhe FL, Pereira AC, Fornazari DH. O Sistema de Informação da Atenção Básica como ferramenta da gestão em saúde. RF0. 2009 [citado 2017 out 18];14(1):77-81. Disponível em: http://seer.upf.br/index.php/ rfo/article/view/675/434Rfo/.

85. Pinto IC, Bulgarelli AF, Gomes TS, Figueiredo LA, Forster AC, Puime A0. Os sistemas de informação em atenção primária como instrumento de gestão em saúde: análise de experiências na Espanha. Cad Saúde Coletiva. 2010 [citado 2017 out 18]; 18(2):291-7. Disponível em: http://www.cadernos.iesc.ufrj.br/ cadernos/images/csc/2010_2/artigos/CSCv18n2_291-297.pdf.

86. Ishfaq M, Lodhi BK. Role of GIS in social sector planning: can developing countries benefit from the examples of Primary Health Care (PHC) planning in Britain? J Community Health. 2012;37(2):372-82. doi: https://doi.org/10.1007/ s10900-011-9454-7.

87. Jonas LT, Rodrigues HC, Resck ZMR.A função gerencial do enfermeiro na Estratégia Saúde da Família: limites e possibilidades. Rev APS. 2011 [citado 2017 out 18];14(1):28-38. Disponível em: http://ojs2.uff.emnuvens.com.br/ aps/article/view/14656.

88. Shaw S, Ashcroft J, Petchey R. Barriers and opportunities for developing sustainable relationships for health improvement: The case of public health and primary care in the UK. Crit Public Health. 2006;16(1):17-88. doi: https://doi. org/10.1080/09581590600602229.

89. Macq J, Martiny P, Villalobos LB, Solis A, Miranda J, Mendez HC, et al. Public purchasers contracting external primary care providers in Central America for better responsiveness, efficiency of health care and public governance: issues and challenges. Health Policy. 20087 Oct 18];87(3):377-88. doi: https://doi. org/10.1016/j.healthpol.2008.01.007.

90. Costa e Silva V, Escoval A, Hortale VA. Contratualização na Atenção Primária à Saúde: a experiência de Portugal e Brasil. Ciênc Saúde Coletiva. 2014;19(8):3593604. doi: https://doi.org/10.1590/1413-81232014198.11822013.

91. Sousa MF, Merchán-Hamann E. Saúde da Família no Brasil: estratégia de superação da desigualdade na saúde? Physis. 2009;19(3):711-29. doi: https:// doi.org/10.1590/S0103-73312009000300009.

92. Castanheira ERL, Nemes MIB, Zarili TFT, Sanine PR, Corrente JE. Avaliação de serviços de Atenção Básica em municípios de pequeno e médio porte no estado de São Paulo: resultados da primeira aplicação do instrumento QualiAB. Saúde Debate. 2014;38(103):679-91. doi: https://doi.org/10.5935/01031104.20140063.

93. Andrade MCl, Castanheira ERL. Cooperação e apoio técnico entre estado e municípios: a experiência do programa articuladores da atenção básica em São Paulo. Saúde Soc. 2011;20(4):980-90. doi: https://doi.org/10.1590/ S01042902011000400015.

94. Nickel DA, Calvo MCM, Natal S, Freitas SFT, Hartz ZMA. Desenvolvimento da capacidade avaliativa na gestão da atenção básica: um estudo de caso exemplar em Santa Catarina, Brasil, de 2008 a 2011. Cad Saúde Pública. 2014;30(4):83950. doi: https://doi.org/10.1590/0102-311X00032413.

95. Santos Neto JA, Mendes AN, Pereira AC, Paranhos LR. Analysis of the Unified Health System funding and expenditure in the municipalities of the "Rota dos Bandeirantes" health region, State of São Paulo, Brazil. Ciênc Saúde Coletiva. 2017;22(4):1269-80. doi: https://doi.org/10.1590/141381232017224.28452016.

96. Lorenzetti J, Lanzoni GMM, Assuiti LFC, Pires DEP, Ramos FRS. Gestão em saúde no Brasil: diálogo com gestores públicos e privados. Texto Contexto Enferm. 2014 June;23(2):417-25. Available from: http://dx.doi.org/10.1590/010407072014000290013

97. Soratto J, Pires DEP, Dornelles S Lorenzetti J. Family health strategy: a technological innovation in health. Texto Contexto Enferm. 2015;24(2):584-92. doi: https://doi.org/10.1590/0104-07072015001572014.

98. Fertonani HP, Pires DEP, Biff D, Scherer MDA. Modelo assistencial em saúde: conceitos e desafios para a atenção básica brasileira. Ciênc Saúde Coletiva. 2015;20(6):1869-78. doi: https://doi.org/10.1590/1413- 
81232015206.13272014.

99. Silva FHC. A atuação dos enfermeiros como gestores em Unidades Básicas de Saúde. Rev Gest Sist Saúde. 2012;1(1):67-82 doi: http://dx.doi.org/10.5585/ rgss.v111.5.

100. Ferreira SRS, Périco LAD, Dias VRGF. The complexity of the work of nurses in primary health care. Rev Bras Enferm. 2018;71(Supl 1):704-9. doi: https://doi. org/10.1590/0034-7167-2017-0471.

101. Miccas FL, Batista, SHSS. Permanent education in health: a review. Rev Saúde Pública. 2014;48(1):170-85. doi: https://doi.org/10.1590/S00348910.2014048004498.

102. Carvalho MN, Gil CRR, Costa EMOD, Sakai MH, Leite SN. Needs and dynamics of thePrimary Healthcareworkforce in Brazil. Ciênc Saúde Coletiva. 2018;23(1):295302. doi: https://doi.org/10.1590/1413-81232018231.08702015.
103. Silva LAA, Soder RM, Petry L, Oliveira IC. Permanent education in primary health care: perception of local health managers. Rev Gaúcha Enferm. 2017;38(1):e58779. doi: https://doi.org/10.1590/1983-1447.2017.01.58779.

104. Martins CC, Waclawovsky AJ. Problemas e desafios enfrentados pelos gestores públicos no processo de gestão em saúde. Rev Gest Sist Saúde. 2015;4(1):1009. Doi: http://dx.doi.org/10.5585/rgss.v4i1.157.

105. Lapão LV, Arcêncio RA, Popolin MP, Rodrigues LBB. The role of Primary Healthcare in the coordination of Health Care Networks in Rio de Janeiro, Brazil, and Lisbon region, Portugal. Ciênc Saúde Coletiva. 2017;22(3):713-24. doi: https://doi.org/10.1590/1413-81232017223.33532016.

106. García-Vera M, Merighi MAB, Conz CA, Silva MH, Jesus MCP, MuñozGonzález LA. Primary health care: the experience of nurses. Rev Bras Enferm. 2018;71(Suppl 1):531-7. doi: https://doi.org/10.1590/0034-7167-2016-0244.
- Autor correspondente:

Denise Elvira Pires de Pires

E-mail: piresdp@yahoo.com
Recebido: 26.07.2018

Aprovado: 12.03.2019 